21

\title{
Оптические свойства гиперосмотических агентов для иммерсионного просветления тканей в терагерцовом диапазоне
}

\author{
(C) Г.Р. Мусина ${ }^{1,2}$, А.А. Гавдуш ${ }^{1,2}$, Н.В. Черномырдин 1, 2, И.Н. Долганова ${ }^{2,3}$, В.Э. Улитко ${ }^{3}$ О.П. Черкасова ${ }^{4,5}$, \\ В.Н. Курлов ${ }^{3}$, Г.А. Командин ${ }^{1}$, И.В. Животовский ${ }^{2}$, В.В. Тучин ${ }^{5,6,7}$, К.И. Зайцев ${ }^{1,2}$ \\ ${ }^{1}$ Институт общей фризики им. А.М. Прохорова РАН, \\ 119991 Москва, Россия \\ ${ }^{2}$ Московский государственный технический университет им. Н.Э. Баумана, \\ 105005 Москва, Россия \\ ${ }^{3}$ Институт фоизики твердого тела РАН, \\ 142432 Черноголовка, Россия \\ ${ }^{4}$ Институт лазерной фризики Сибирского отделения РАН, \\ 630090 Новосибирск, Россия \\ ${ }^{5}$ Национальный исследовательский Томский государственный университет, \\ 634050 Томск, Россия \\ ${ }^{6}$ Саратовский национальный исследовательский государственный университет имени Н.Г. Чернышевского, \\ 410012 Саратов, Россия \\ ${ }^{7}$ Институт проблем точной механики и управления РАН, \\ 410028 Саратов, Россия \\ e-mail: guzel-musina12@mail.ru
}

Поступила в редакцию 18.02.2020 г.

В окончательной редакции 03.03.2020 г.

Принята к публикации 28.03.2020 г.

\begin{abstract}
Измерены спектры пропускания наиболее распространенных гиперосмотических агентов, таких как чистые глицерин, пропиленгликоль (ПГ), диметилсульфоксид (ДМСО), полиэтиленгликоль (ПЭГ) с молекулярными массами 200, 300, 400 и $600 \mathrm{Da}$ и их водные растворы, а также водных растворов сахарозы, глюкозы, фруктозы, декстрана 40 и 70. Эксперименты проводились с помощью ТГц импульсного спектрометра с вакуумированным измерительным отсеком для уменьшения влияния паров воды на спектральные измерения. Восстановлены диэлектрические характеристики гиперосмотических агентов в спектральном диапазоне от 0.1 до $2.5 \mathrm{THz}$ и построена зависимость амплитудного коэффициента поглощения от концентрации рассмотренных агентов на частоте $0.5 \mathrm{THz}$. Полученные результаты позволяют выбрать оптимальные агенты для иммерсионного оптического просветления в ТГц диапазоне.
\end{abstract}

Ключевые слова: терагерцовые технологии, терагерцовая импульсная спектроскопия, диэлектрическая спектроскопия, иммерсионное оптическое просветление, просветляющие агенты, дегидратирующие агенты.

DOI: $10.21883 /$ OS.2020.07.49576.65-20

\section{Введение}

В наше время терагерцовая (ТГц) спектроскопия находит своё применение во все большем количестве областей: в системах неразрушающего контроля материалов [1,2], обеспечения безопасности [3], оценке качества химической и фармацевтической продукции [4] и др. При этом возможности ТГц технологий привлекают все большее внимание в биомедицинской сфере [5], где становятся основой создания новых диагностических методов для таких заболеваний, как злокачественные образования кожи [6-8], молочной железы [9], кишечника [10], мозга [11-14]. Несмотря на достигнутый прогресс ряд вопросов, связанных с эффективными методами генерации и детектирования излучения ТГц диапазона, остаются в той или иной степени нерешенными [15], в частности можно отметить:

- высокую стоимость и громоздкость ТГц оборудования [16];
- отсутствие как волноводов, так и волокон для доставки ТГц излучения к труднодоступным органам и тканям [17-19];

- низкое пространственное разрешение ТГц оптических систем, обусловленное дифракционным пределом и большой длиной волны излучения [20-22];

- малую глубину проникновения ТГц излучения в биологические ткани, обусловленную сильным поглощением ТГц волн свободной и связанной водой в тканях [16].

Для решения вышеперечисленных задач требуются значительные исследовательские и инженерные усилия. В данной работе мы рассмотрим один из возможных методов решения последней задачи, а именно увеличение глубины проникновения ТГц излучения в ткани. Снижать поглощение ТГц излучения, обусловленное высоким содержанием воды в биотканях, возможно с 
Перечень используемых ОПА

\begin{tabular}{c|c|c}
\hline Агенты & $\%$ & Производитель \\
\hline Полиэтиленгликоль (ПЭГ) 200 & $10-100 \%$ & Нижнекамскнефтехим, Россия \\
Полиэтиленгликоль (ПЭГ) 300 & $10-100 \%$ & Sigma-Aldrich, Германия \\
Полиэтиленгликоль (ПЭГ) 400 & $10-100 \%$ & Нижнекамскнефтехим, Россия \\
Полиэтиленгликоль (ПЭГ) 600 & $10-100 \%$ & ООО „Норкем“, Россия \\
Диметилсульфоксид (ДМСО) & $10-100 \%$ & SpektrChem, Pocсия \\
Пропиленгликоль (ПГ) & $10-100 \%$ & Chemical Line Co. Ltd, Pocсия \\
Глицерин & $10-100 \%$ & SpektrChem, Pocсия \\
Сахароза & $10-90 \%$ & PanReac, Испания \\
Глюкоза & $10-90 \%$ & Hungarna KFT, Венгрия \\
Фруктоза & $10-90 \%$ & PanReac, Испания \\
Декстран 40 & $10-60 \%$ & AppliChem, Германия \\
Декстран 70 & $10-50 \%$ & AppliChem, Германия
\end{tabular}

помощью различных физических и химических воздействий, оказываемых на ткани, среди них:

- заморозка [23,24];

- дегидратация [25];

- лиофилизация [26];

- парафинизация [27];

- фиксация в формалине [28];

- иммерсионное оптическое просветление [29-35].

Данные методы, кроме последнего, занимают много времени, требуют сложной подготовки и не могут быть использованы in vivo. Более того, некоторые из этих способов вызывают необратимые структурные изменения в тканях.

Иммерсионное оптическое просветление - это один из перспективных методов управления оптическими свойствами биотканей в ТГц диапазоне частот, основанный на замещении свободной воды в тканях на гиперосмотический просветляющий агент [36]. В процессе иммерсионного просветления происходит диффузия агента в исследуемую ткань и замещение им воды, что снижает поглощение ТГц излучения. Несмотря на значительный потенциал данного метода для ТГц биофотоники, его исследования в основном ограничиваются видимым и инфракрасным диапазонами [36-46], лишь отдельные работы посвящены методам иммерсионного оптического просветления в ТГц области спектра [29-35]. Нерешенной проблемой остается выбор оптимальных агентов, поскольку отсутствует база данных с ТГц диэлектрическими характеристиками обсуждаемых веществ.

Для создания базы данных оптически-просветляющих агентов (ОПА) в ТГц диапазоне мы выполнили ТГц импульсную спектроскопию в режиме пропускания следующих веществ: чистых глицерина, пропиленгликоля (ПГ), диметилсульфоксида (ДМСО), полиэтиленгликоля (ПЭГ) с молекулярными массами 200, 300, 400 и $600 \mathrm{D}$, их водных растворов, а также водных растворов сахарозы, глюкозы, фруктозы, декстрана 40 и 70. Были восстановлены диэлектрические характеристики данных агентов в спектральном диапазоне от 0.1 до $2.5 \mathrm{THz}$ и построена зависимость амплитудного коэффициента поглощения от концентрации рассмотренных агентов на частоте $0.5 \mathrm{THz}$. Полученные результаты позволяют выбрать оптимальные агенты для иммерсионного оптического просветления в ТГц диапазоне.

\section{Оптически-просветляющие агенты}

В таблице представлены агенты, изученные в данной работе. Для приготовления водных растворов использовалась деионизованная вода. Жидкие препараты растворяли до необходимой концентрации, смешивая определенный объем вещества с необходимым объемом воды. Порошки взвешивали и добавляли определенный объем воды.

\section{Экспериментальная установка}

Измерение ТГц диэлектрических свойств агентов было выполнено на оригинальном ТГц импульсном спектрометре в режиме регистрации проходящего сигнала (рис. $1, a)$. Данная система позволяет исследовать спектральные характеристики образца в диапазоне частот от 0.2 до $4.0 \mathrm{THz}$ с максимальным спектральным разрешением около $0.015 \mathrm{THz}$. При работе с кюветой для измерения жидкостей установка обеспечивает спектральный рабочий диапазон от 0.25 до $2.5 \mathrm{THz}$ из-за дифракции ТГц пучка на апертуре кюветы, френелевских потерь на его поверхности, а также поглощения ТГц пучка в объеме среды.

Генерация и детектирование широкополосных ТГц импульсов в данной системе производится в фотопроводящих антеннах 1,2 ; коллимация и фокусировка ТГц пучков осуществляется при помощи внеосевых параболических зеркал с золотым напылением 3, 4. Измерения проводились с вакуумированием измерительного отсека ТГц импульсного спектрометра, что позволяет исключить влияние паров воды в атмосфере на результаты (это влияние особенно критично на высоких 


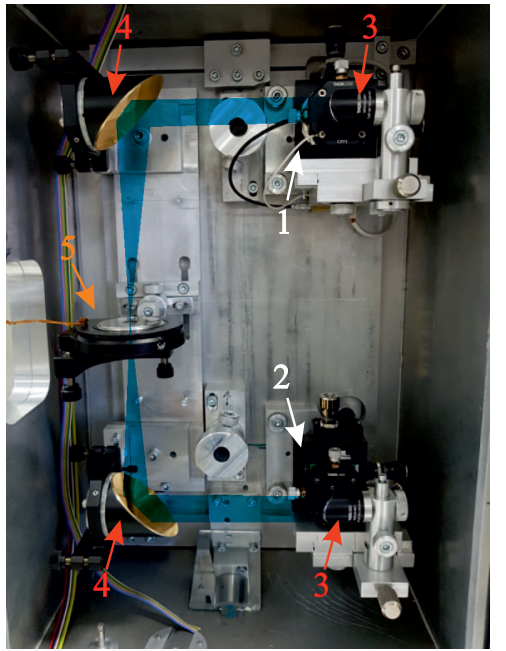

$a$

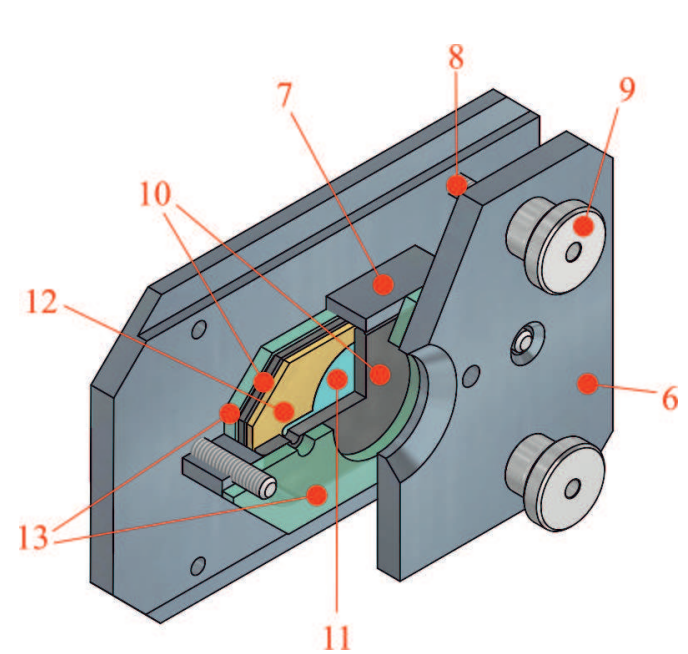

$b$

Рис. 1. (a) ТГц импульсный спектрометр в режиме регистрации проходящего сигнала: 1,2 - фотопроводящая антенна для генерации и детектирования ТГц пучков; 3, 4 - внеосевые параболические зеркала с золотым напылением для коллимации и фокусировки ТГц пучков; 5 - держатель для образца; (b) разработанная кювета для измерения диэлектрических характеристик агентов в ТГц импульсном спектрометре с вакуумированием измерительного отсека. 6,7 - крышка и основание, соединенные четырьмя винтами 8 и гайками 9. Два окна 10, изготовленных из высокоомного кремния. Регулирование толщины образца 11 происходит с помощью латунных разделителей 12. Для обеспечения герметичности кюветы были использованы резиновые прокладки 13.

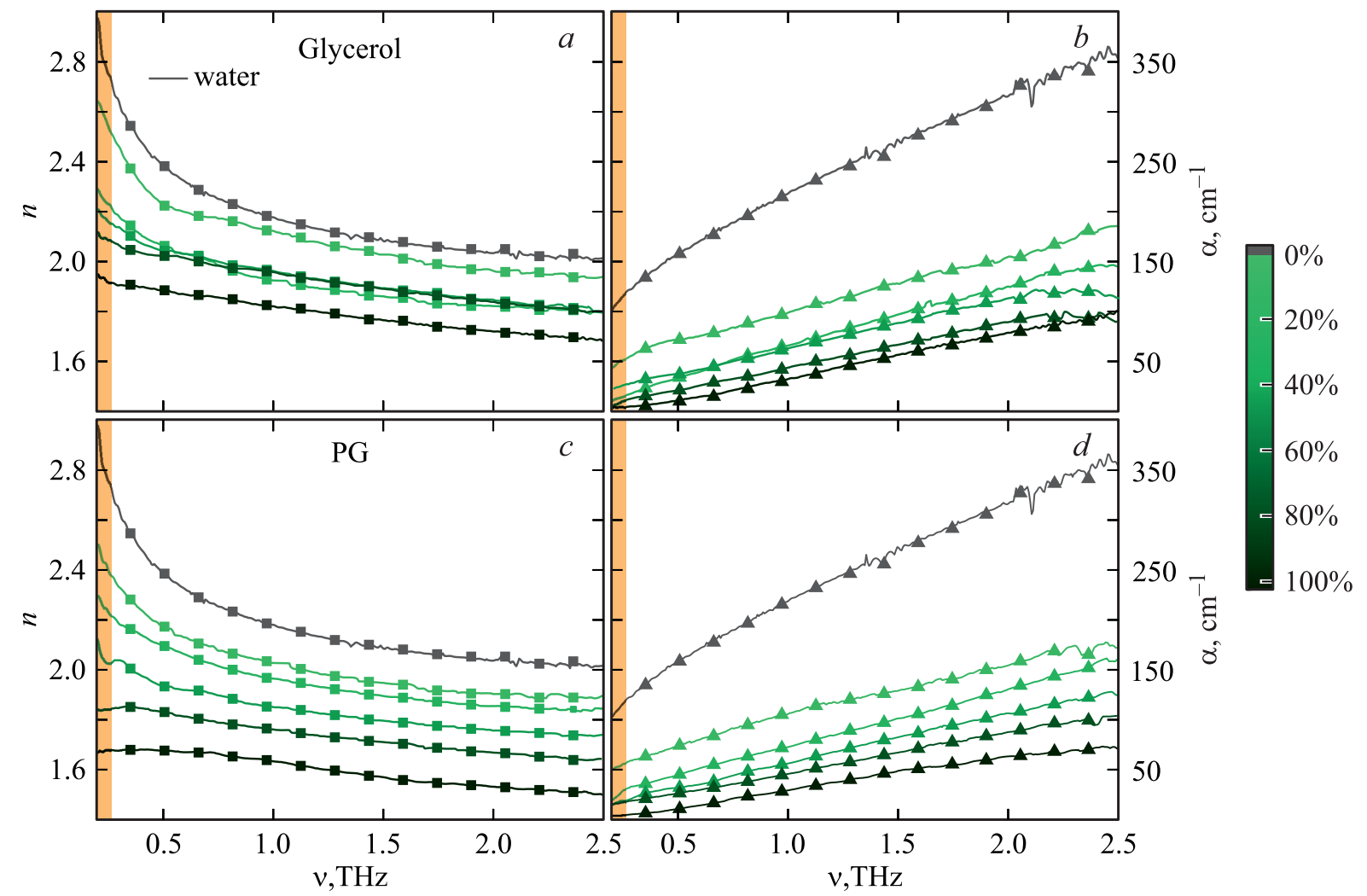

Рис. 2. Показатель преломления $n(a, c)$ и амплитудный коэффициент поглощения $\alpha(b, d)$ воды $(0 \%)$ и водных растворов глицерина $(a, b)$ и ПГ $(c, d)$ в концентрации от $20 \%$ до $100 \%$ с шагом $20 \%$ (см. шкалу концентраций справа). 


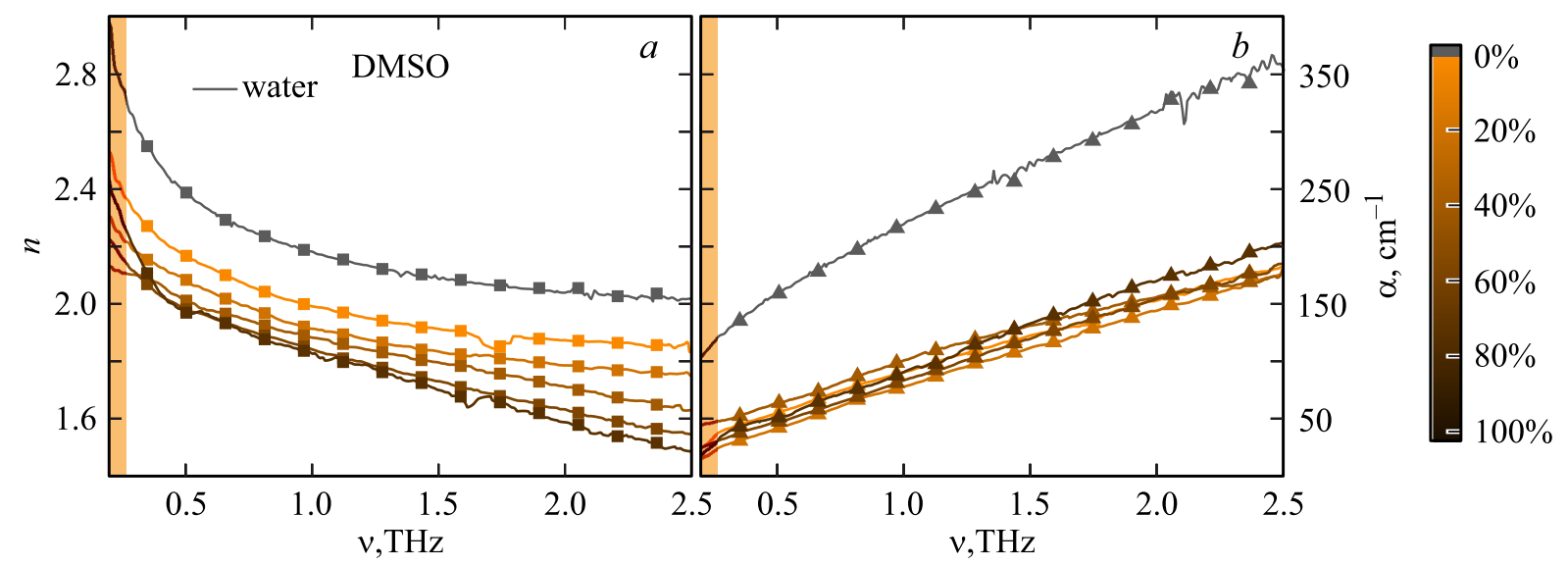

Рис. 3. Показатель преломления $n(a)$ и амплитудный коэффициент поглощения $\alpha(b)$ воды $(0 \%)$ и водных растворов ДМСО в концентрации от 20\% до $100 \%$ с шагом $20 \%$ (см. шкалу концентраций справа).

частотах $>1.5 \mathrm{THz})$ и соответственно максимально расширить рабочий спектральный диапазон. Как показано на рис. $1, b$, разработанная кювета для измерения жидкостей состоит из двух металлических частей: крышки 6 и основания 7 , соединенных четырьмя винтами 8 и гайками 9. Два окна, изготовленных из высокоомного кремния 10, толщиной $2 \mathrm{~mm}$ расположены внутри кюветы. Регулирование толщины образца 11 происходит с помощью латунных разделителей 12 толщиной 100, 200, $300 \mu \mathrm{m}$. Для обеспечения герметичности кюветы были использованы резиновые прокладки 13.

\section{Восстановление ТГц диэлектрических свойств}

Мы использовали алгоритм восстановления ТГц диэлектрических свойств, описанный ранее в работах $[34,47]$. В эксперименте регистрируются опорный сигнал и сигнал образца. Опорный сигнал проходит через пустую кювету, входное и выходное окна которой плотно прижаты друг к другу. Сигнал образца регистрируется для трехслойной среды, когда исследуемый образец жидкости расположен между кремниевыми окнами. Поскольку кремниевые окна имеют значительную толщину при определенном выборе аподизационного фильтра, можно не учитывать переотражений в них ТГц импульса. После упрощения математические модели детектируемых сигналов могут быть представлены в виде:

$$
\begin{gathered}
\tilde{E}_{s}=\tilde{E}_{0} \tilde{T}_{01} \tilde{T}_{12} \tilde{T}_{21} \tilde{T}_{10} \frac{\tilde{P}_{1}^{2}\left(l_{1}\right) \tilde{P}_{2}\left(l_{2}\right)}{\tilde{P}_{0}^{2}\left(l_{1}\right) \tilde{P}_{0}\left(l_{2}\right)} \frac{1-\left(\tilde{P}_{2}^{2} \tilde{R}_{22}^{2}\right)^{N}}{1-\tilde{P}_{2}^{2} \tilde{R}_{21}^{2}}, \\
\tilde{E}_{r}=\tilde{E}_{0} \tilde{T}_{01} \tilde{T}_{10} \frac{\tilde{P}_{1}^{2}\left(l_{1}\right)}{\tilde{P}_{0}^{2}\left(l_{1}\right)}
\end{gathered}
$$

где индекс 0 соответствует пустому пространству, 1 окнам кюветы (высокоомный кремний или пластик), 2 - образцу, $N$ - число учитываемых внутри образца переотражений ТГц сигнала; $\tilde{E}_{s}$ - комплексная амплитуда сигнала, прошедшего через образец; $\tilde{E}_{r}-$ комплексная амплитуда опорного сигнала; $\tilde{T}, \tilde{R}-$ комплексные амплитудные коэффициенты пропускания и отражения поверхности для нормального падения излучения соответственно определяются формулами Френеля; $\tilde{P}-$ комплексный коэффициент пропускания среды, определяется законом Бугера-Ламберта-Бера.

Восстановление комплексного показателя преломления, а следовательно, и комплексной диэлектрической проницаемости исследуемых образцов осуществляется путем минимизации функционала ошибки:

$$
\tilde{n}(v)=\operatorname{argmin}\{\Phi(v, \tilde{n}(v))\},
$$

где

$$
\Phi(v, \tilde{n}(v))=\left(\begin{array}{c}
\left|\tilde{H}_{\text {exp }}(v)\right|-\left|\tilde{H}_{t h}(v, \tilde{n}(v))\right| \\
\left|\varphi\left(\tilde{H}_{\text {exp }}(v)\right)-\varphi\left(\tilde{H}_{t h}(v, \tilde{n}(v))\right)\right|
\end{array}\right),
$$

$\tilde{H}_{\text {exp }}(v)$ и $\tilde{H}_{t h}(v, \tilde{n}(v))$ - экспериментальная и теоретическая передаточные функции; |...| и $\varphi(\ldots)-$ операторы модуля и фазы.

Передаточная функция представляет собой отношение спектров сигнала образца и опорного сигнала,

$$
\tilde{H}_{\exp }=\frac{\tilde{E}_{s, \exp }}{\tilde{E}_{r, \exp }}
$$

$\tilde{E}_{s, \exp }$ и $\tilde{E}_{r, \exp }-$ комплексные амплитуды сигнала, прошедшего через образец и опорного сигнала соответственно.

Теоретическая передаточная функция с учетом полученных математических моделей сигналов примет вид:

$$
\tilde{H}_{t h}=\tilde{T}_{12} \tilde{T}_{21} \frac{\tilde{P}_{2}\left(l_{2}\right)}{\tilde{P}_{0}\left(l_{2}\right)} \frac{1-\left(\tilde{P}_{2}^{2} \tilde{R}_{21}^{2}\right)^{N}}{1-\tilde{P}_{2}^{2} \tilde{R}_{21}^{2}} .
$$




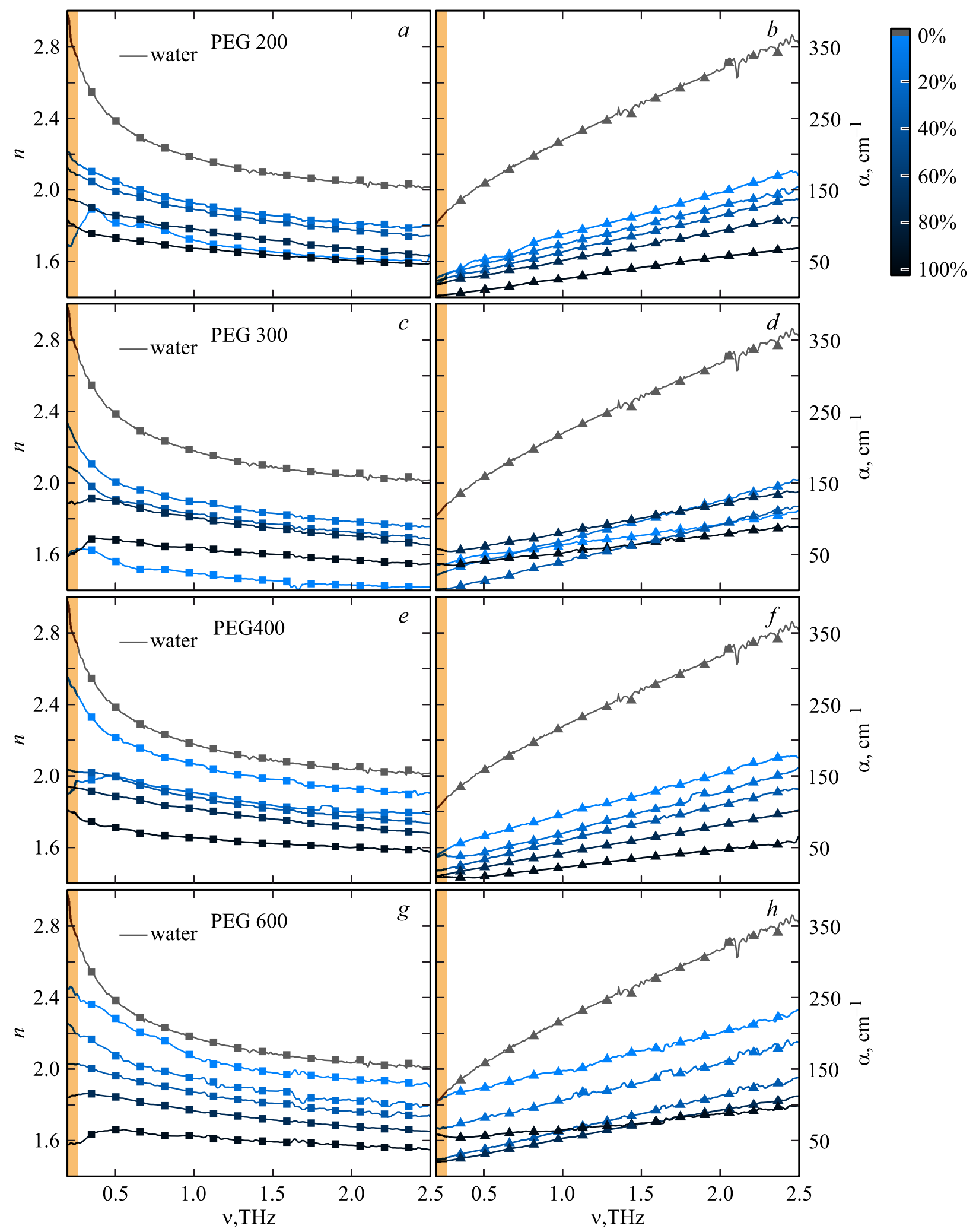

Рис. 4. Показатель преломления $n(a, c, e, g)$ и амплитудный коэффициент поглощения $\alpha(b, d, f, h)$ воды $(0 \%)$ и водных растворов ПЭГ $200(a, b), 300(c, d), 400(e, f), 600(g, h)$ в концентрации от 20\% до 100\% с шагом 20\% (см. шкалу концентраций справа). 


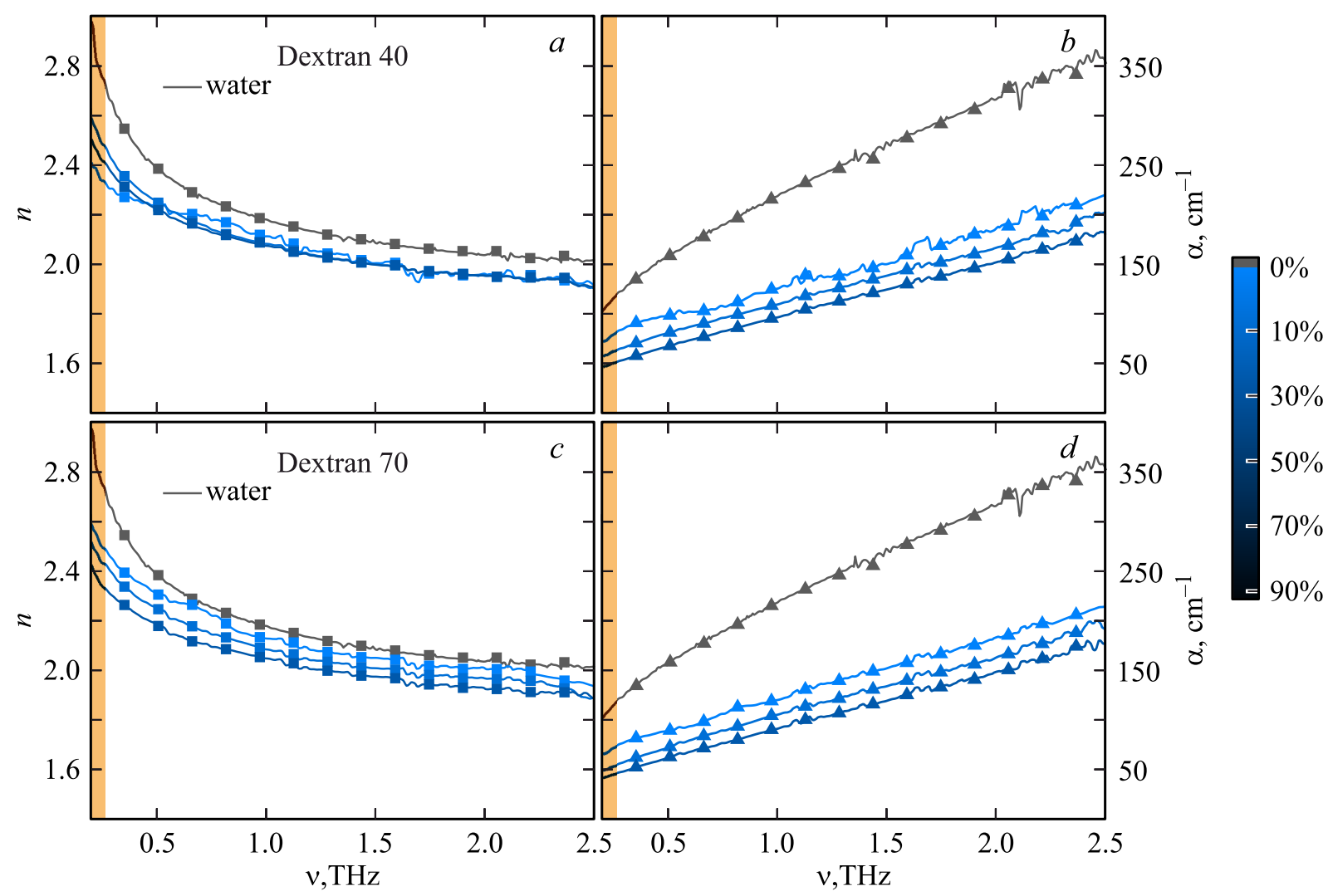

Рис. 5. Показатель преломления $n(a, c)$ и амплитудный коэффициент поглощения $\alpha(b, d)$ воды $(0 \%)$ и водных растворов декстрана $40(a, b), 70(c, d)$ в концентрации от $10 \%$ до 50\% с шагом $20 \%$ (см. шкалу концентраций справа).

Взаимодействие ТГц излучения с границами раздела кюветы описывается формулами Френеля для нормального падения; они определяют пропускание и отражение ТГц луча на границе раздела между $m$-й и $k$-й средами соответственно:

$$
\tilde{T}_{m, k}=\frac{2 \tilde{n}_{m}}{\tilde{n}_{m}+\tilde{n}_{k}}, \quad \tilde{R}_{m, k}=\frac{\tilde{n}_{m}-\tilde{n}_{k}}{\tilde{n}_{m}+\tilde{n}_{k}},
$$

где $\tilde{n}_{m}$ и $\tilde{n}_{k}$ - комплексные показатели преломления двух сред. В свою очередь, закон Бугера-ЛамбертаБера описывает фазовую задержку и поглощение ТГц излучения в $q$-й среде

$$
\tilde{P}\left(\tilde{n}_{q}, l_{q}\right)=\exp \left(-i \frac{2 \pi v}{c} \tilde{n}_{q} l_{q}\right),
$$

где $\tilde{n}_{q}$ и $l_{q}$ - комплексный показатель преломления и геометрическая длина этой среды.

\section{Результаты}

На основе сигналов ТГц импульсного спектрометра была проведена оценка ТГц оптических свойств гиперосмотических агентов. На рис. 2-6 показаны амплитудные коэффициенты поглощения и показатели преломления всех рассматриваемых агентов в частотном диапазоне от
0.2 до $2.5 \mathrm{THz}$. Для сравнения полученных результатов на графиках также приведены амплитудный коэффициент поглощения и показатель преломления воды.

Агенты были разделены на следующие классы [48] и обозначены определенным цветом на рисунках: рис. 2, зеленый - спирты и производные, рис. 3, оранжевый сульфоксиды и амиды, рис. 4,5 - синий, полимеры, рис. 6 - красный, сахара и сахарные спирты.

На рис. 2-4 приведены результаты измерения характеристик растворов с концентрациями от 20\% до $100 \%$. На рис. 5 и 6 приведены значения измеренных характеристик для растворов сухих веществ. Показаны результаты для растворов сахаров (рис. 6) с концентрациями от $10 \%$ до $90 \%$ и для растворов декстрана 40 и 70 (рис. 5) с концентрациями от $10 \%$ до 50\%. Выбор концентраций для декстрана обусловлен тем, что получение более концентрированных растворов данных веществ затруднительно. Все растворы измерены с шагом в $20 \%$.

На спектрах (рис. 2-6) слева выделена область низкочастотного диапазона $0.2-0.25 \mathrm{THz}$, где ожидаются искажения данных, вызванные дифракцией. Стоит отметить, что относительная погрешность в рассматриваемом спектральном диапазоне для показателя преломления равна 0.02, для амплитудного коэффициента 


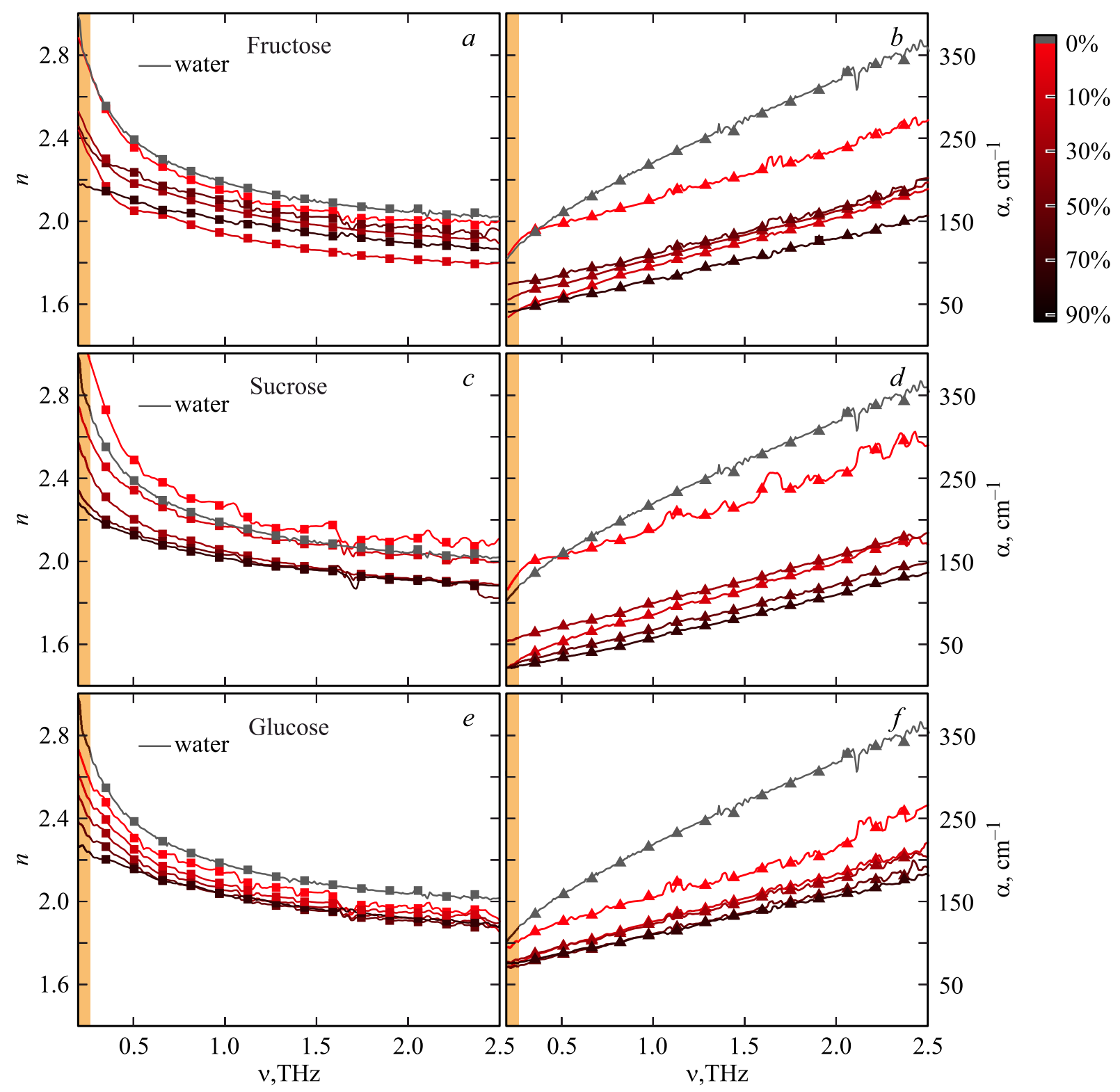

Рис. 6. Показатель преломления $n(a, c, e)$ и амплитудный коэффициент поглощения $\alpha(b, d, f)$ воды $(0 \%)$ и водных растворов сахаров: фруктозы $(a, b)$, сахарозы $(c, d)$, глюкозы $(e, f)$ в концентрации от $10 \%$ до $90 \%$ с шагом $20 \%$ (см. шкалу концентраций справа).

поглощения - 0.096. Полученные результаты хорошо согласуются с ранее полученными данными [34,35].

На рис. 7 показана зависимость амплитудного коэффициента поглощения от концентрации агента на частоте $0.5 \mathrm{THz}$. Мы использовали разделение агентов по вышеперечисленным классам. На рисунке видно уменьшение амплитудного коэффициента поглощения с увеличением концентрации раствора. Его неравномерный характер может быть связан с погрешностями измерений или особенностями агентов, что требует дальнейшего изучения.

На выбор оптимального агента влияет не только коэффициент поглощения, но и скорость диффузии агента в ткань [35]. Чем выше скорость диффузии, тем быстрее происходит оптическое просветление. Поэтому в зависимости от области применения просветления в ТГц биофотонике важно делать выбор агента по величине его коэффициента поглощения и скорости диффузии в ткани.

\section{Заключение}

В данной работе приведены результаты ТГц импульсной спектроскопии в режиме пропускания ОПА. Были исследованы: чистые и водные растворы глицерина, ПГ, ДМСО, ПЭГ с молекулярными массами 200, 300, 400 и $600 \mathrm{Da}$, а также водные растворы сахарозы, глюко- 

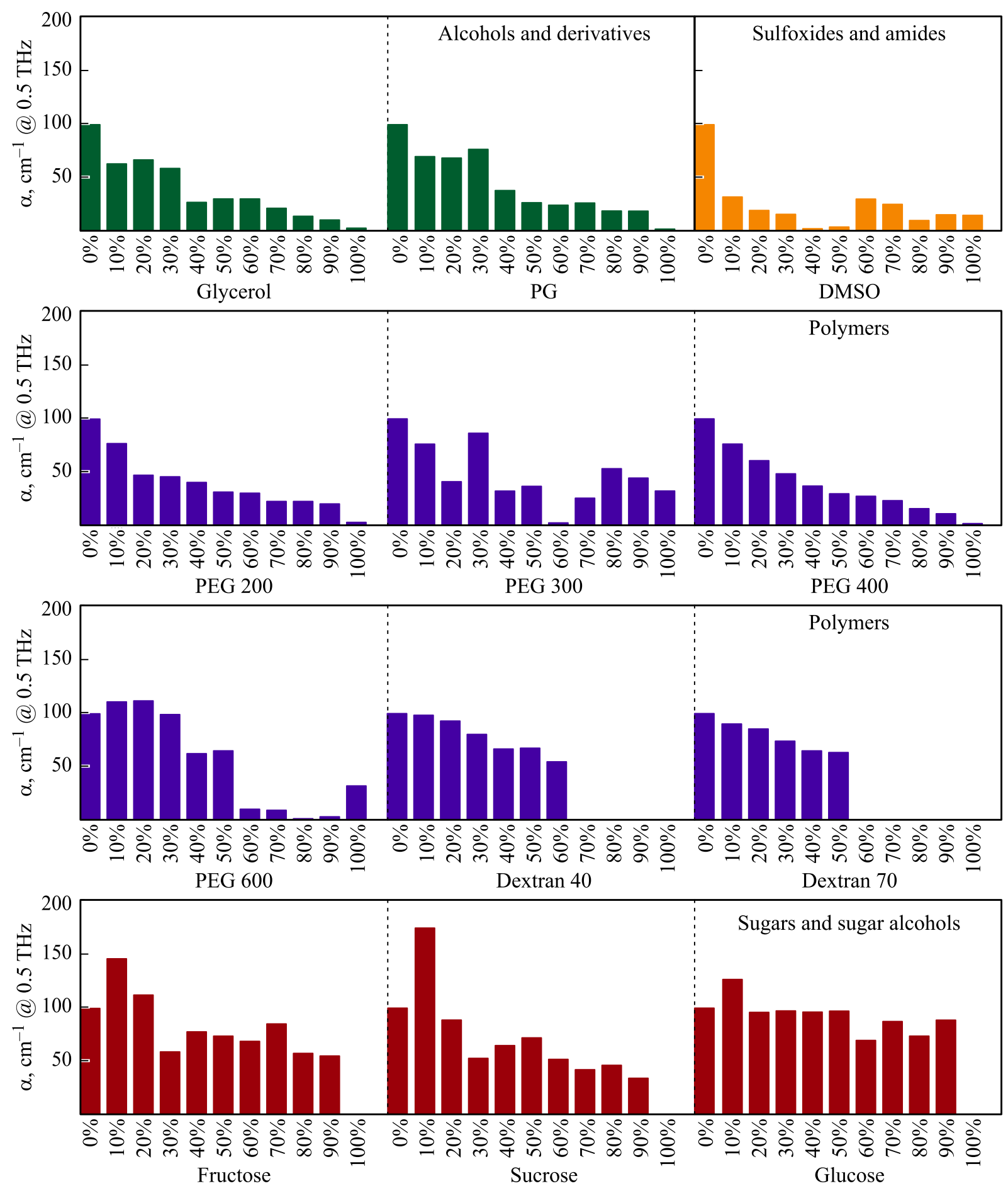

Рис. 7. Зависимости амплитудного коэффициента поглощения от концентрации рассмотренных агентов на частоте $0.5 \mathrm{THz}$

зы, фруктозы, декстрана 40 и 70. Были восстановлены диэлектрические характеристики данных агентов в спектральном диапазоне от 0.1 до $2.5 \mathrm{THz}$, построена зависимость амплитудного коэффициента поглощения от концентрации для каждого агента на частоте $0.5 \mathrm{THz}$. Полученные результаты позволяют выбрать оптимальные агенты для иммерсионного оптического просветления в ТГц диапазоне.

\section{Финансирование работы}

Разработка кюветы И.Н. Долгановой финансирована Грантом Президента РФ для государственной поддержки молодых российских ученых - кандидатов наук МK-2541.2019.8. Анализ данных поглощения растворов агентов с различной концентрацией Н.В. Черномырдиным и В.В. Тучиным поддержано 
Российским фондом фундаментальных исследований (РФФИ), проект № 19-32-50075. Выбор просветляющих агентов выполнен В.В. Тучиным в рамках проекта РФФИ № 18-52-16025 НЦНИЛ_а.

\section{Список литературы}

[1] Yakovlev E.V., Zaytsev K.I., Dolganova I.N., Yurchenko S.O. // IEEE Trans. terahertz science and technology. 2015. V. 5. N 5. P. 810-816. doi 10.1109/TTHZ.2015.2460671

[2] Yakovlev E.V., Zaytsev K.I., Chernomyrdin N.V., Gavdush A.A., Zotov A.K., Nikonovich M.Y., Yurchenko S.O. // Proc. SPIE. 2016. V. 9899. P. 98990 W. doi $10.1117 / 12.2227321$

[3] Dolganova I.N., Zaytsev K.I., Metelkina A.A., Karasik V.E., Yurchenko S.O. // Rev. Sci. Instr. 2015. V. 86. N 11. P. 113704. doi 10.1063/1.4935495

[4] Zeitler J.A., Taday P.F., Newnham D.A., Pepper M., Gordon K.C., Rades T. // J. Pharm. Pharmacol. 2007. V. 59. N 2. P. 209-223. doi 10.1211/jpp.59.2.0008

[5] Smolyanskaya O.A., Chernomyrdin N.V., Konovko A.A., Zaytsev K.I., Ozheredov I.A., Cherkasova O.P., Nazarov M.M., Guillet J.-P., Kozlov S.A., Kistenev Y.V., Coutaz J.-L., Mounaix P., Vaks V.L., Son J.-H., Cheon H., Wallace V., Shkurinov A.P., Tuchin V.V. // Progr. Quantum Electron. 2018. V. 62. P. 1.77. doi 10.1016/j.pquantelec.2018.10.001

[6] Woodward R.M., Wallace V.P., Pye R.J., Cole B.E., Arnone D.D., Linfield E.H., Pepper M. // J. Investigative Dermatology. 2003. V. 120. N 1. P. 72-78. doi 10.1046/j.1523-1747.2003.12013.x

[7] Zaytsev K.I., Gavdush A.A., Chernomyrdin N.V, Yurchenko S.O. // IEEE Trans. terahertz sci. technol. 2015. V. 5. N 5. P. 817-827. doi 10.1109/TTHZ.2015.2460677

[8] Zaytsev K.I., Kudrin K.G., Karasik V.E., Reshetov I.V, Yurchenko S.O. // Appl. Phys. Lett. 2015. V. 106. N 5. P. 053702. doi 10.1063/1.4907350

[9] Fitzgerald A.J., Wallace V.P., Jimenez-Linan M., Bobrow L., Pye R.J., Purushotham A.D., Arnone D.D. // Radiology. 2006. V. 239. N 2. P. $533-540$. doi 10.1148/radiol.2392041315

[10] Reid C.B., Fitzgerald A., Reese G., Goldin R., Tekkis P., O'Kelly P.S., Pickwell-MacPherson E., Gibson A.P., Wallace V.P. // Physics in Medicine and Biology. 2011. V. 56. N 14. P. 4333-4353. doi 10.1088/0031-9155/56/14/008

[11] Oh S.J., Kim S.-H., Ji Y. Bin, Jeong K., Park Y., Yang J., Park D.W., Noh S.K., Kang S.-G., Huh Y.-M., Son J.-H., Suh J.-S. // Biomed. Optics Express. 2014. V. 5. N 8. P. 2837. doi 10.1364/BOE.5.002837

[12] Gavdush A.A., Chernomyrdin N.V., Malakhov K.M., Beshplav S.-I.T., Dolganova I.N., Kosyrkova A.V., Nikitin P.V., Musina G.R., Katyba G.M., Reshetov I.V., Cherkasova O.P., Komandin G.A., Karasik V.E., Potapov A.A., Tuchin V.V., Zaytsev K.I. // J. Biomed. Optics. 2019. V. 24. N 02. P. 027001. doi 10.1117/1.JBO.24.2.027001

[13] Chernomyrdin N.V., Gavdush A.A., Beshplav S.-I.T., Malakhov K.M., Kucheryavenko A.S., Katyba G.M., Dolganova I.N., Goryaynov S.A., Karassik V.E., Spector I.E., Kurlov V.N., Yurchenko S.O., Komandin G.A., Potapov A.A., Tuchin V.V., Zaytsev K.I. // Proc. SPIE. 2018. V. 10716. P. 107160 S. doi $10.1117 / 12.2316302$
[14] Chernomyrdin N.V., Dolganova I.N., Beshplav S.-I.T., Aleksandrova P.V., Musina G.R., Malakhov K.M., Nikitin P.V., Kosyr'kova A.V., Komandin G.A., Reshetov I.V., Potapov A.A., Tuchin V.V., Zaytsev K.I. // Proc. SPIE. 2019. V. 10864. P. 1086406. doi 10.1117/12.2506600

[15] Lee Y.-S. // Principles of Terahertz Science and Technology. N.Y.: Springer, 2009. 347 p. doi 10.1007/978-0-387-09540-0

[16] Smolyanskaya O., Chernomyrdin N., Konovko A., Zaytsev K., Ozheredov I., Cherkasova O., Nazarov M., Guillet J.-P., Kozlov S., Kistenev Y., Coutaz J.-L., Mounaix P., Vaks V., Son J.-H., Cheon H., Wallace V., Feldman Y., Popov I., Yaroslavsky A., Shkurinov A., Tuchin V. // Progr. Quantum Electronics. 2018. V. 62. P. 1-77. doi 10.1016/j.pquantelec.2018.10.001

[17] Zaytsev K., Katyba G., Kurlov V., Shikunova I., Karasik V., Yurchenko S. // IEEE Trans. Terahertz Sci. Technol. 2016. V. 6. N 4. P. 576-582. doi 10.1109/TTHZ.2016.2555981

[18] Katyba G., Zaytsev K., Chernomyrdin N., Shikunova I., Komandin G., Anzin V., Lebedev S., Spektor I., Karasik V., Yurchenko S., Reshetov I., Kurlov V., Skorobogatiy M. // Adv. Opt. Mater. 2018. V. 6. N 22. P. 1800573. doi 10.1002/adom.201800573

[19] Katyba G., Zaytsev K., Dolganova I., Shikunova I., Chernomyrdin N., Yurchenko S., Komandin G., Reshetov I., Nesvizhevsky V., Kurlov V. // Progr. Crystal Growth and Characterization of Materials. 2018. V. 64. N 4. P. 133-151. doi 10.1016/j.pcrysgrow.2018.10.002

[20] Fan B., Neel V., Yaroslavsky A. // Lasers in Surgery and Medicine. 2017. V. 49. N 3. P. 319-326. doi $10.1002 / \mathrm{lsm} .22552$

[21] Chernomyrdin N., Frolov M., Lebedev S., Reshetov I., Spektor I., Tolstoguzov V., Karasik V., Khorokhorov A., Koshelev K., Schadko A., Yurchenko S., Zaytsev K. // Rev. Sci. Instr. 2017. V. 88. N 1. P. 014703. doi 10.1063/1.4973764

[22] Chernomyrdin N., Kucheryavenko A., Kolontaeva G., Katyba G., Dolganova I., Karalkin P., Ponomarev D., Kurlov V., Reshetovza I., Skorobogatiy M., Tuchin V., Zaytsev K. // Appl. Phys. Lett. 2018. V. 113. N 11. P. 111102. doi 10.1063/1.5045480

[23] Hoshina H., Hayashi A., Miyoshi N., Miyamaru F., Otani C. // Appl. Phys. Lett. 2009. V. 94. N 12. P. 123901. doi 10.1063/1.3106616

[24] Sim Y., Ahn K.-M., Park J., Park C.-S., Son J.-H. // IEEE Transactions on terahertz sci. technol. 2013. V. 17. N 4. P. 368-373. doi 10.1109/JBHI.2013.2252357

[25] He Y., Liu K., Au C., Sun Q., Parrott E., PickWellMacPherson E. // Physics in Medicine and Biology. 2017. V. 62. N 23. P. $8882-8893$. doi 10.1088/1361-6560/aa8ebe

[26] Png G., Choi J., Ng B.-H., Mickan S., Abbott D., Zhang X.C. // Physics in Medicine and Biology. 2008. V. 53. N 13. P. 3501-3517. doi 10.1088/0031-9155/53/13/007

[27] Meng K., Chen T.-N., Chen T., Zhu L.-G., Liu Q., Li Z., Li F., Zhong S.-C., Li Z.-R., Feng H., Zhao J.H. // J. Biomed. Optics. 2014. V. 19. N 7. P. 077001. doi 10.1117/1.JBO.19.7.077001

[28] Sun Y., Fischer B., Pickwell-MacPherson E. // J. Biomed. Optics. 2009. V. 14. N 6. P. 064017. doi 10.1117/1.3268439

[29] Oh S., Kim S.-H., Jeong K., Park Y., Huh Y.-M., Son J.H., Suh J.-S. // Optics Express. 2013. V. 21. N 18. P. 21299-21305. doi 10.1364/OE.21.021299 
[30] Kolesnikov A., Kolesnikova E., Kolesnikova K., Tuchina D., Popov A., Skaptsov A., Nazarov M., Shkurinov A., Terentyuk A., Tuchin V. // Physics of Wave Phenomena. 2014. V. 22. N 3. P. $169-176$. doi 10.3103/S1541308X14030029

[31] Kolesnikov A., Kolesnikova E., Tuchina D., Terentyuk A., Nazarov M., Skaptsov A., Shkurinov A., Tuchin V. // Proc. SPIE. 2014. V. 9031. P. 90310D. doi 10.1117/12.2052226

[32] Kolesnikov A., Kolesnikova E., Popov A., Nazarov M., Shkurinov A., Tuchin V. // Quant. Electron. 2014. V. 44. N 7. P. 633-640. doi 10.1070/QE2014v044n07ABEH015493

[33] Smolyanskaya O., Schelkanova I., Kulya M., Odlyanitskiy E., Goryachev I., Tcypkin A., Grachev Y., Toropova Y., Tuchin V. // Biomed. Optics Express. 2018. V. 9. N 3. P. 1198-1215. doi 10.1364/BOE.9.001198

[34] Musina G., Dolganova I., Malakhov K., Gavdush A., Chernomyrdin N., Tuchina D., Komandin G., Chuchupal S., Cherkasova O., Zaytsev K., Tuchin V. // Proc. SPIE. 2018. V. 10800. P. 108000F. doi 10.1117/12.2324473

[35] Musina G., Gavdush A., Tuchina D., Dolganova I., Komandin G., Chuchupal S., Smolyanskaya O., Cherkasova O., Zaytsev K., Tuchin V. // Proc. SPIE. 2019. V. 11065. P. 110651Z. doi $10.1117 / 12.2526168$

[36] Tuchin V.V. // Tissue Optics: Light Scattering Methods and Instruments for Medical Diagnosis: 3rd Edition. Bellingham: SPIE Press. 2007. 882 p. doi 10.1117/3.1003040

[37] Genina E., Bashkatov A., Sinichkin Y., Yanina I., Tuchin V. // J. Biomed. Photon. Eng. 2015. V. 1. N 1. P. 22-58. doi 10.18287/jbpe-2015-1-1-22

[38] Bashkatov A., Berezin K., Dvoretskiy K., Chernavina M., Genina E., Genin V., Kochubey V., Lazareva E., Pravdin A., Shvachkina M., Timoshina P., Tuchina D., Yakovlev D., Yakovlev D., Yanina I., Zhernovaya O., Tuchin V. /I J. Biomed. Optics. 2018. V. 23. N 9. P. 091416. doi 10.1117/1.JBO.23.9.091416

[39] Mao Z., Zhu D., Wen X., Han Z. // J. Biomed. Optics. 2008. V. 13. N 2. P. 021104. doi $10.1117 / 1.2892684$

[40] Tuchina D., Genin V., Bashkatov A., Genina E., Tuchin V. // Opt. Spectrosc. 2016. V. 120. N 1. P. 36-45. doi 10.1134/S0030400X16010215

[41] Oliveira L., Carvalho M., Nogueira M., Tuchin V. // J. Biomed. Optics. 2015. V. 20. N 5. P. 051019. doi 10.1117/1.JBO.20.5 .051019

[42] Guo X., Guo Z.Y., Wei H.J., Yang H.Q., He Y.H., Xie S.S., Wu G.Y., Zhong H.Q., Li L.Q., Zhao Q.L. // Laser Phys. 2010. V. 20. N 9. P. $1849-1855$. doi 10.1134/S1054660X10170032

[43] Carvalho S., Gueiral N., Nogueira E., Henrique R., Oliveira L., Tuchin V.V. // J. Biomed. Optics. 2017. V. 22. N 9. P. 091506. doi 10.1117/1.jbo.22.9.091506

[44] Liu P., Huang Y., Guo Z., Wang J., Zhuang Z., Liu S. // J. Biomed. Optics. 2013. V. 18. N 2. P. 020507. doi 10.1117/1.jbo.18.2.020507

[45] Zhao Q., Wei H., He Y., Ren Q., Zhou C. // J. Biophotonics. 2014. V. 7. N 11-12. P. 938-947. doi $10.1002 /$ jbio. 201300141

[46] Zhao Q.L., Si J.L., Guo Z.Y., Wei H.J., Yang H.Q., Wu G.Y., Xie S.S., Li X.Y., Guo X., Zhong H.Q. // Laser Phys. Lett. 2010. V. 8. N 1. P. 71-77. doi 10.1002/lapl.201010081

[47] Gavdush A., Ulitko V., Musina G., Dolganova I., Chernomyrdin $N$., Kurlov $V$., Komandin G., Tuchin V., Zaytsev K. // Proc. SPIE. 2019. V. 11060. P. $110601 \mathrm{G}$. doi $10.1117 / 12.2527649$
[48] Oliveira L., Tuchin V. // The Optical Clearing Method: A New Tool for Clinical Practice and Biomedical Engineering. Switzerland: Springer Briefs in Physics. 2019. 155 p. doi 10.1007/978-3-030-33055-2 